\title{
KNOWLEDGE AND SKILLS RELATED TO ACTIVE OPTICAL SENSORS IN THE BODY OF KNOWLEDGE FOR EARTH OBSERVATION AND GEOINFORMATION (EO4GEO BOK)
}

\author{
(in alphabetical order) \\ C. Dubois ${ }^{1, * \dagger}$, B. Jutzi ${ }^{2}$, M. Olijslagers ${ }^{3}$, C. Pathe ${ }^{1}$, C. Schmullius ${ }^{1}$, M. A. Stelmaszczuk-Górska ${ }^{1, \dagger}$, D. Vandenbroucke ${ }^{3}$, M. $^{2}$ \\ Weinmann ${ }^{2}$ \\ ${ }^{1}$ Dept. for Earth Observation, University of Jena, Germany - (clemence.dubois,c.pathe, c.schmullius, m.stelmas)@uni-jena.de \\ ${ }^{2}$ Institute of Photogrammetry and Remote Sensing, Karlsruhe Institute of Technology, Germany - (boris.jutzi, \\ martin.weinmann)@kit.edu \\ ${ }^{3}$ Spatial Applications Division Leuven, KU Leuven, Belgium - (marc.olijslagers, danny.vandenbroucke)@kuleuven.be
}

\section{Commission V, WG V/1}

\begin{abstract}
KEY WORDS: Earth Observation, Geoinformation, Active optical sensors, LiDAR, Body of Knowledge, Concepts, Skills
\end{abstract} Definition

\begin{abstract}
:
The field of Earth Observation (EO) and Geoinformation (GI) is gaining more and more importance due to the increasing number of data and data processing algorithms to respond even more accurately to a variety of challenges in many application areas. In order to follow recent activities and align the exponential evolution of datasets and recent processing trends with market and academic training requirements, the EO*GI sector needs an updated and new definition of knowledge and skills. To this goal, a specific body of knowledge for Earth Observation and Geoinformation (EO4GEO BoK) is currently being implemented with the aim of providing interconnected concepts and job-oriented skills. One novelty of the BoK is to include topics related to Earth Observation, in particular to introduce the different sensors used in this field. Active optical sensors are at the crossroad between Earth Observation and closerange photogrammetry and have not been described in any other existing BoK. This paper introduces therefore the part of the EO4GEO BoK that is dedicated to active optical sensors. Such systems are used in various job-oriented applications such as archeology, mobile mapping, indoor or outdoor, or for surveying purposes, just to name a few. The structure of this part of the BoK is explained and specific descriptions and relationships between the identified concepts are given. Finally, the skills acquired by completing these BoK concepts are presented and discussed in terms of how they can be used in a professional context or in the definition of job-oriented learning paths.
\end{abstract}

\section{INTRODUCTION}

The rapidly and exponentially growing data quantity in the field of Earth Observation and Geoinformation (EO*GI), associated with an open policy for most of these data and new algorithms, triggers a paradigm shift in learning and knowledge transfer (Sudmanns et al., 2019). In order to take into account the latest approaches and respond to upcoming challenges in EO*GI education and the labour market (Hofer et al., 2020), the project "Towards an innovative strategy for skills development and capacity building in the space geo-information sector supporting Copernicus user uptake" (EO4GEO; http://www.eo4geo.eu/) defines new and specific knowledge and skills for the creation of curricula and training actions.

One of the principal goals of EO4GEO is to bring together experts from different specific areas of the $\mathrm{EO}$ GI domain to create an exhaustive and comprehensive, structured knowledge that can be used to define and develop training actions or curricula in the EO*GI sector. This structured knowledge is based on the creation of a Body of Knowledge (BoK) that serves as a reference tool for education and training activities (Stelmaszczuk-Górska et al., 2020a).

In this paper, we describe the conception of a specific part of the BoK dedicated to active optical sensors. The choice of this particular domain is driven by the large variety of sensors and platform types, providing a wide range of applications, both at global (i.e., spaceborne) and local scale (i.e., close-range photogrammetry). This represents a challenge for the BoK development, as different levels of information have to be created and organized in the BoK in such a way that all information is available but not redundant, and based on it, optimal learning paths for training actions and curricula can be defined.

\section{BODY OF KNOWLEDGE}

Beside giving important definitions and an overview of existing bodies of knowledge in the geoinformation sector, this section highlights the novelty of the EO4GEO BoK.

\subsection{Definition}

A body of knowledge $(\mathrm{BoK})$ is a structured representation of knowledge in a particular domain. It includes a set of concepts, as well as their descriptions and corresponding activities or skills that can be used by members of a discipline to guide their education and work practice (DiBiase et al., 2006). It can be represented as a mind map or node diagram, where concepts are interconnected via relationships building the ontology. The concepts are usually a theory, a method or a technology, associated with their respective knowledge description. Activities can be learning outcomes if the BoK should be used primarily in an academic context, or skills if the BoK is used in both academic and professional context.

\footnotetext{
$\dagger$ Both authors contributed equally
} 
A BoK can be used as reference for a particular professional domain, as it defines what knowledge and competences are necessary to be able to perform specific job tasks.

\subsection{State-of-the-Art}

Many domains and sectors have developed their BoK, e.g., PMBOK is a BoK related to Project Management (PMI, 2017), while the DAMA DMBOK is a BoK describing the field of Data Management (DAMA International, 2017).

In the geoinformation (GI) sector, a first comprehensive documentation of the geographic information science domain and its associated technologies (GIS\&T) has been provided in form of a BoK by the University Consortium for Geographic Information Science (UCGIS). All the concepts described were published as a publicly available book in digital format (DiBiase et al., 2006). The concepts described in the GIS\&T BoK are grouped in ten knowledge areas and regularly updated to the latest developments in the field, so that they now include, among others, artificial intelligence approaches and tools, big data visualization techniques, but also the description of specific softwares or libraries. The further development of this original BoK was pursued separately in the United States and in Europe. The American version focused on the academic content, where definitions of knowledge areas and concepts are presented in the form of textbooks without any interconnections and relationships between the concepts. Each core concept is published together with its subconcepts in the form of a scientific peer-reviewed document. Instead of skills definition, learning outcomes and instructional assessment questions are the focus of this academic BoK.

Each topic is described in the form of scientific, peer-reviewed documents and uniquely identified using a DOI and a permanent URL (Wilson, 2020; http://gistbok.ucgis.org). This BoK represents a more academic approach.

The European version focused on a web-based semantic representation of the GIS\&T BoK and its relationships, developed by Ahearn et al. (2013) and resulted in the Geographic Information Need to Know (GI-N2K) BoK (Vandenbroucke and Vancauwenberghe, 2016). Hierarchical relationships such as "is subconcept of", "is super-concept of", "is pre-requisite of", "is post-requisite of" and non-hierarchical relationships such as "is similar to" allow learners or curricula builders to navigate throughout the BoK and acquire missing competencies or skills. The GI-N2K BoK has been reported to be particularly useful for the definition of module catalogues and study paths (Reinhardt, 2014).

Concerning the domain of active optical sensors, only a few attempts have been made to structure this knowledge into a BoK format. The GIS\&T BoK presents a LiDAR concept, focusing mainly on the different types of data sources and data formats. Greenfeld (2012) and Bethel (2011) suggested to include LiDAR concepts as part of an active sensing or imagery super-concept within a surveying body of knowledge endorsed by the International Federation of Surveyors (FIG). Although the structure and concepts of the surveying body of knowledge have been thoroughly defined (Greenfeld and Potts 2008, Greenfeld 2010 and Greenfeld 2011), further development of the BoK content is not available.

\subsection{EO4GEO Body of Knowledge}

The EO4GEO BoK, which is currently being developed, is an extended and revised version of the European GI-N2K BoK (Vandenbroucke et al., 2019). It aims at adding new topics from the EO sector and updating the GI part, building a comprehensive knowledge of both EO and GI (Stelmaszczuk-Górska et al., 2020a), and creating a bridge to potential other BoKs, such as a surveying BoK. Additionally, it focuses on expanding user uptake by adding a business-oriented perspective (Hofer et al., 2020).

The current implementation of the EO4GEO BoK is organized in seven working groups (WGs) (Stelmaszczuk-Górska et al., 2020a), three of them on updating topics related to geoinformation, three on new Earth Observation topics and one on thematic applications. In the first publicly available version of the EO4GEO BoK (Stelmaszczuk-Górska et al., 2020b), the EO4GEO BoK has 907 concepts interconnected by 1129 relationships, defining 1875 skills. The part dedicated to active optical sensors is within the WG dedicated to "Platforms, Sensors and Digital Imagery". This WG identified 85 concepts, from types of remote sensing platforms and sensors to data formats, through mission planning.

The EO4GEO BoK is the backbone of the skill strategy for the EO*GI domain. Different tools have been developed to exploit the BoK content, which can be used, among others, for the development of curricula or learning paths, as well as for jobrelated activities (Aguilar-Moreno et al., 2020). It creates a framework for the preparation of training activities (Krieger, 2020). The BoK for $\mathrm{EO} * \mathrm{GI}$ is developed using the Living Textbook environment developed by the University of Twente (Lemmens et al., 2018). The Living Textbook (LTB) allows viewing, commenting and modifying the content of the BoK and was used for the implementation of the concepts related to active optical sensors.

In this paper, we show the creation and implementation of the EO4GEO BoK part dedicated to active optical sensors, as it represents a novelty that cannot be found in any other BoK and is a highly relevant topic at the crossroad between GI, EO and surveying. Furthermore, the EO4GEO BoK aims at strengthening the business perspective of the BoK. In this way, the definition of specific tasks and the transcription of these into knowledge and skills plays a key role. Here we will present the process of defining the active optical sensors part of the EO4GEO BoK.

\section{ACTIVE OPTICAL SENSORS IN THE EO4GEO BOK}

The taxonomy of active optical sensors related topics can be found in different textbooks (Jutzi, 2015; Jutzi et al., 2016; Kramer, 2002). However, the knowledge structure and

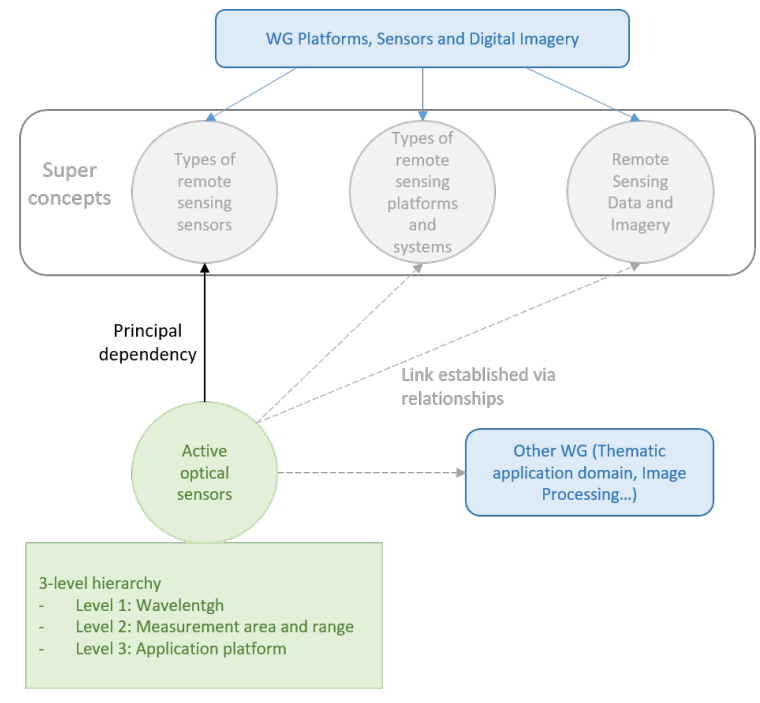

Figure 1. Integration of the active optical sensors within the BoK 
definitions are not always consistent as they mainly depend on the thematic scope of the textbooks (e.g. sensors, platforms, data formats, data processing, data handling, electric engineering or image processing). As integrated to the WG "Platforms, Sensors and Digital Imagery", the related concepts could be defined through a number of taxonomies. In the LTB, this WG has been divided into three main super-concepts: "Types of remote sensing sensors", "Types of remote sensing platforms and systems", and "Remote Sensing Data and Imagery". The first super-concept focuses on the identification and definition of different sensor types, their properties and operating principles. The second super-concept focuses on the platform types, i.e. ground, airborne or spaceborne, and the last super-concept is dedicated to principles and properties of data and digital imagery. Whilst showing some relationships with the other two super-concepts, the concepts of active optical sensors and their subconcepts have been assigned to the first super-concept "Types of remote sensing sensors" (Figure 1). The choice was driven by the different types of active optical sensors and their specific operating and measuring principles that are, for an application and job-driven point of view, more relevant than the type of platforms they are situated on. Especially, some sensors, such as laser scanners, can be found on different types of platforms. Likewise, data processing cannot be unambiguously identified. The different types of active optical sensors, together with their principles of operation and measurement, have been described from their possible application's point of view. The selected taxonomy of the different active optical sensors as presented in the BoK was defined after Jutzi et al. (2016).

The concepts are structured in a three-level hierarchy, whereby the first level of hierarchy (Level-1) organizes the sensors by wavelength, the second level by measurement area or range (Level-2), and the third level by specific characteristics or application platforms (Level-3). Especially for Level-2, a distinction has been made between ranging and scanning devices, as not only the geometry but also the radiometry measured by some sensors plays an important role in their application.

\subsection{Knowledge}

While other existing BoKs containing LiDAR descriptions focus on the different data formats (Keranen and Kolvoord, 2019), this part of the EO4GEO BoK focuses first on data collection devices, i.e., sensors and acquisition platforms, data formats being considered in a different part of the BoK (Stelmaszczuk-Górska et al., 2020a). Therefore, the organization of knowledge in this field started from grouping different types of sensors depending on the type of information recorded by the device (time-of-flight or range, in 1D or 2D) and their application. This decision was in line with the overarching concept "Types of remote sensing sensors". In addition, the lower concept levels define subconcepts that correspond to the different types of platforms, e.g., ground, airborne, spaceborne, which in turn is in line with the second core concept "Types of remote sensing platforms".

Knowledge related to active optical sensors has been gathered for the EO4GEO BoK based on the review of scientific literature by experts in the field (Stelmaszczuk-Górska et al, 2020a) and described according to the prepared manuals (e.g., Lemmens et al., 2020a). For the first publicly available version of the BoK, which is presented here, the knowledge of the identified concepts was provided as a short ( $<50$ words) or long (250-500 words) description. The latter was prepared for the core concepts. Short descriptions that were prepared for the remaining concepts will be systematically replaced with long ones.

Specific requirements were defined for the descriptions of the knowledge in concepts. Each description should give an overview of the specific knowledge in relation with the core concept. In the case of the LiDAR concepts, for example, the difference between the different sensors should be highlighted, and at least one field of application should be mentioned. The application areas can be additionally defined by establishing relationships with other working groups of the EO4GEO BoK, i.e. "Thematic Application Domains" or "Image Processing and Analysis" which include concepts such as "Monitor the Forest", "DEM generation" or "Point Clouds". No particular sensor model or trading mark should be stated, as those become obsolete over time.

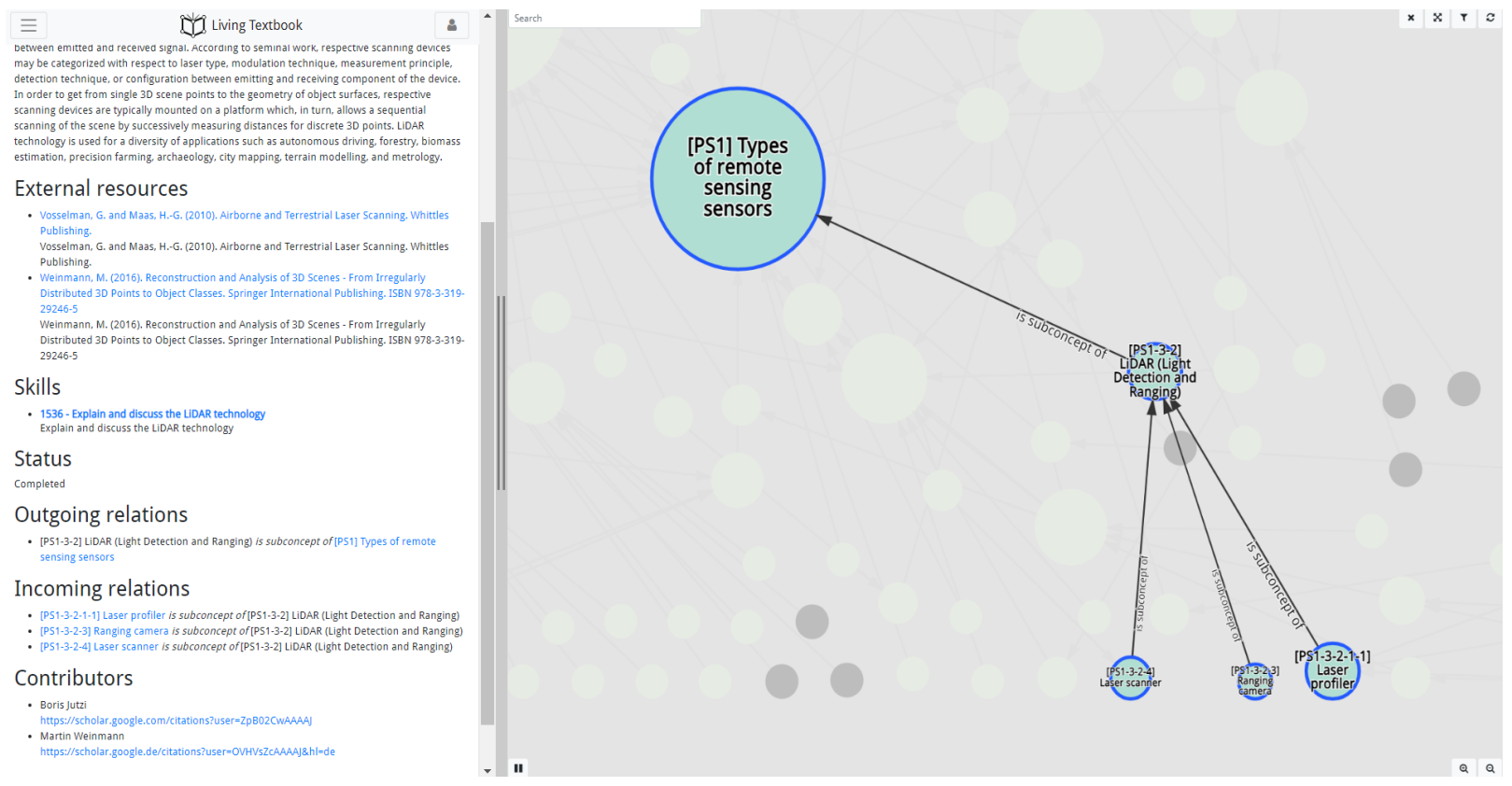

Figure 2. Living Text Book page of the LiDAR concept with corresponding ontology 


\subsection{Ontology}

The ontology principle, as being a verbal and formal representation of a set of concepts and the relationships between them in a specific subject area, plays a key role in the EO4GEO BoK. This means that each concept is described in relation to another, creating one complete and coherent base of knowledge in the field of EO and GI, which can be used as a basis for creating curricula and training programs. The interconnections of the BoK concept are built using a specific ontology implemented in the Living TextBook Environment (LTB) (Lemmens et al., 2018). The interface of the LTB combines a concept map with a text based window containing the description of each concept, including its description, references, contributors, skills, and relationships (categorised in incoming and outcoming relations). Currently, the following types of relationships are available:

- Subconcept (a concept on a lower granularity level)

- Prerequisite (one concept needs to be known to understand the other)

- $\quad$ Similarity (one concept is similar to another).

An example of the implemented ontology in the LTB is given in Figure 2.

\subsection{Skills}

The skills of the EO4GEO BoK aim at adding a business perspective to the BoK. This is to optimize the process of providing people with the skills needed by the market.

Whilst the term "learning outcome" is usually used in the learning process, EO4GEO BoK uses the term "skills", as it describes both learning outcomes useful for academia and the competences or ability to complete the task required by the industry.

The skills are combinations of verbs and a concept-relevant statement based on the title of the corresponding BoK concept. The verbs are grouped into a six classes taxonomy as defined in Hofer et al. (2020). They range from passive verbs ("remember", "understand") to higher-level active verbs such as "evaluate" and "create".

\section{KNOWLEDGE AND SKILLS RELATED TO ACTIVE OPTICAL SENSORS}

This section shows an excerpt of the EO4GEO BoK dedicated to active optical sensors. The structure and relationships between the concepts is explained, their description is given, and corresponding skills are listed.

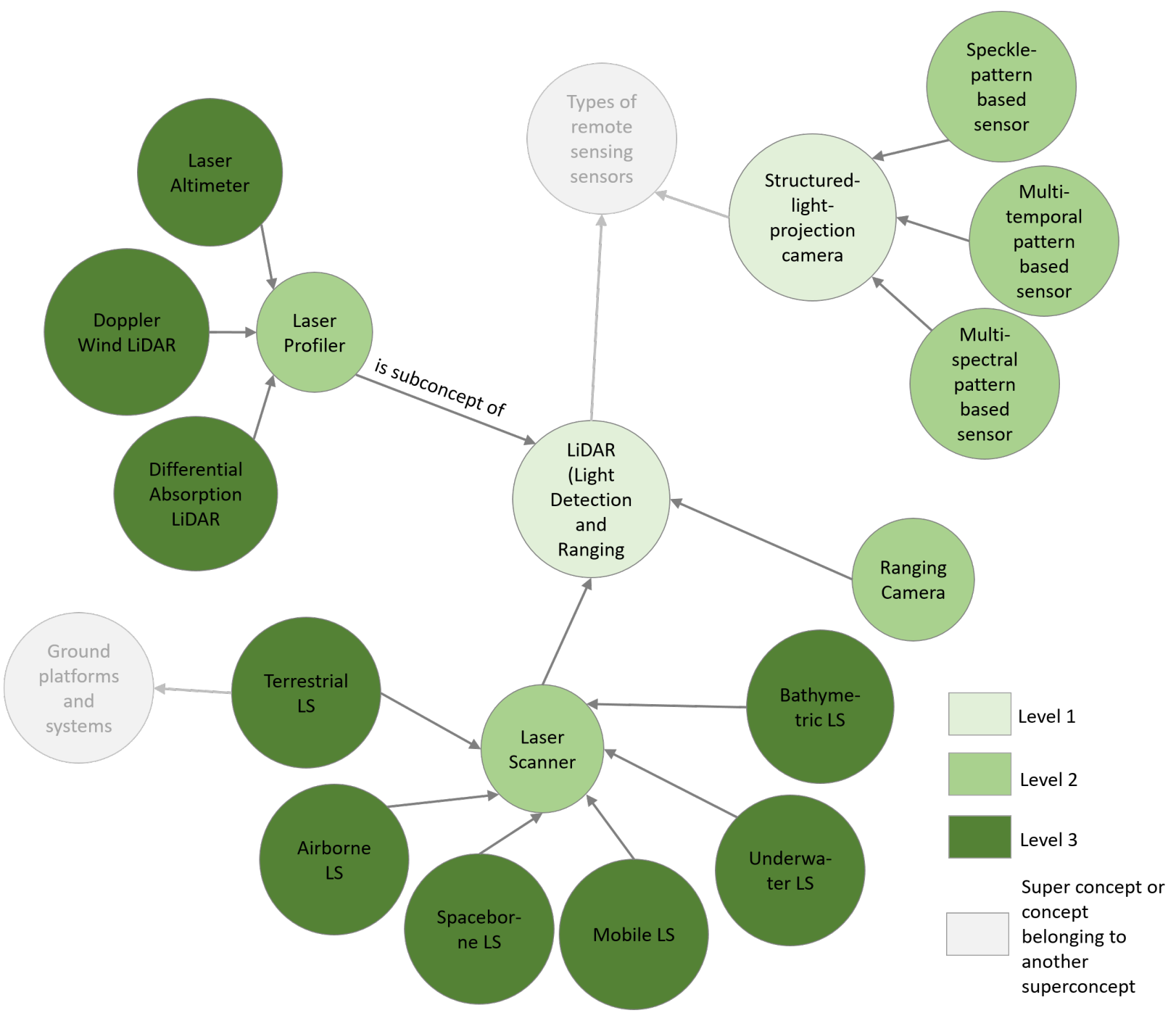

Figure 3. Schematic representation of the EO4GEO BoK ontology of the active optical sensors 


\subsection{Structure of the Concepts}

Two main sensor types have been identified for "active optical sensors", organized by the wavelength they work with. This work focused therefore on the description of those two main concepts, "LiDAR" and "Structured-light-projection camera", and their subconcepts. A total of 17 related concepts has been identified and described in a three-layer structure, following and completing the original categorisation by Jutzi et al. (2016).

The relation between the concepts is shown in Figure 3, where an arrow pointing to a higher-level concept means "is subconcept of". In the example in Figure 3 it should be read as: "Laser profiler is a subconcept of LiDAR". The concept "LiDAR" itself is a subconcept of "Types of remote sensing sensors", which will not be mentioned further here. The subconcept "Terrestrial Laser Scanning" is also related to a subconcept of another main concept "Ground platforms and systems", thus linking to the superconcept "Types of remote sensing platforms" (see Section 3).

\subsection{Knowledge Description}

This section gives an excerpt of the concept descriptions of active optical sensors, as they can be found in the EO4GEO BoK, i.e. organized by knowledge level as described in Section 3.1. The corresponding references are indicated at the end of each concept.

\section{Level-1 concepts - organized by wavelength}

\section{LiDAR (Light Detection and Ranging)}

The main idea of LiDAR (Light Detection and Ranging) technology is based on actively scanning the scene by involving a device which emits electromagnetic radiation in the form of modulated laser light.

Generally, such scanning devices illuminate a scene with modulated laser light and analyze the backscattered signal. More specifically, laser light is emitted by the scanning device and transmitted to an object. At the object surface, the laser light is partially reflected and, finally, a certain amount of the laser light reaches the receiver unit of the scanning device. The measurement principle is therefore of great importance as it may be based on different signal properties such as amplitude, frequency, polarization, time, or phase.

Many scanning devices are based on measuring the time $t$ between emitting and receiving a laser pulse, i.e., the respective time-of-flight, and exploiting the measured time $t$ in order to derive the distance $r$ between the scanning device and the respective 3D scene point. Alternatively, a range measurement $r$ may be derived from phase information by exploiting the phase difference $\Delta \varphi$ between emitted and received signal. According to seminal work, respective scanning devices may be categorized with respect to laser type, modulation technique, measurement principle, detection technique, or configuration between emitting and receiving components of the device.

In order to get from single 3D scene points to the geometry of object surfaces, respective scanning devices are typically mounted on a platform which, in turn, allows a sequential scanning of the scene by successively measuring distances for discrete $3 \mathrm{D}$ points.

LiDAR technology is used for a diversity of applications such as autonomous driving, forestry, biomass estimation, precision farming, archaeology, city mapping, terrain modelling, and metrology (Vosselman and Maas, 2010; Weinmann, 2016).

\section{Structured-light-projection camera}

A structured-light-projection camera emits active optical radiation in the form of a coded structured light pattern in the visible or infrared spectrum, or electromagnetic radiation in the form of modulated laser light. Via the projected pattern, particular labels are assigned to 3D scene points which, in turn, may easily be decoded in images when imaging the scene and the projected pattern with a camera. The procedure reminds of conventional stereo processing, where corresponding features have to be extracted from a pair of stereo images to derive the spatial information. In contrast, such synthetically generated features allow to robustly establish feature correspondences, and the respective $3 \mathrm{D}$ coordinates may easily and reliably be recovered via triangulation. Generally, techniques based on the use of structured light patterns may be classified depending on the pattern codification strategy (Salvi et al., 2004; Dal Mutto et al., 2012; Weinmann, 2016; Geng, 2011).

\section{Level-2 concepts - organized by measurement area and range}

\section{Laser profiler}

Laser profilers measure 1D range profiles and operate in different environments, like spaceborne, airborne and indoor. Most of them operate top-down on flying platforms, but as well bottomup is possible, e.g. in meteorology for cloud monitoring (Rees, 2013; Kramer, 2002).

\section{Laser scanner}

Laser scanners capture data by successively considering points on a discrete, regular (typically spherical) raster, and recording the respective geometric and radiometric information. There are different types of laser scanners depending on their application and the platform on which they are mounted: spaceborne, airborne, terrestrial, mobile, underwater, bathymetric (Bleier et al., 2019; Mandlburger and Jutzi, 2019; Weinmann et al., 2015).

\section{Ranging camera}

By a ranging camera the simultaneous capturing of range measurements in the form of a range image for an extended area of dynamical 3D applications is given. Applications are building surveillance, traffic monitoring, and driver assistance (Dal Mutto et al., 2012).

\section{Speckle-pattern based sensor}

Speckle-pattern based sensors operate with spatial neighborhood codification strategies to exploit a unique pattern. The label associated to a pixel is derived from the spatial pattern distribution within its local neighborhood. Thus, labels of neighboring pixels share information and provide an interdependent coding. Representing one of the most popular devices based on structured light projection, the Microsoft

Kinect exploits an RGB camera, an infrared (IR) camera, and an IR projector. The IR projector projects a known structured light pattern in the form of a random but unique speckle dot pattern onto the scene. As IR camera and IR projector form a stereo pair, the pattern matching in the IR image results in a raw disparity image which, in turn, is read out as a depth image (Dal Mutto et al., 2012)

\section{Multi-temporal pattern based sensor}

A multi-temporal (sequential) binary coding uses black and white stripes to form a sequence of projection patterns for each point on the surface of the object. A binary coding technique is very reliable and less sensitive to the surface characteristics, since only binary values exist in all pixels. Thus, each pixel may be assigned a codeword consisting of its illumination value across the projected patterns. The respective patterns may, for instance, be based on binary codes or Gray codes and phase shifting. To achieve high spatial resolution, a large number of sequential 
patterns need to be projected. All objects in the scene have to remain static. The entire duration of $3 \mathrm{D}$ image acquisition may be longer than a practical 3D application allows for. These sensors are utilized in industrial environment (Geng, 2011).

\section{Multi-spectral pattern based sensor}

For a multi-spectral pattern based sensor, various continuously varying color patterns to encode the spatial location information are utilized (Geng, 2011).

\section{Level-3 concepts - organized by specific characteristics and applications}

\section{Laser altimeter}

Laser altimeters historically were the first active sensing devices used on airborne platforms, measuring range information in form of single distances. Nowadays, they are still found on low-cost platforms like drones to determine the flight altitude (Kramer, 2002; Rees, 2013).

\section{Doppler Wind Lidar}

Cloud-Aerosol Lidar with Orthogonal Polarization (e.g. CALIOP) is a two-wavelength polarization-sensitive LiDAR that provides high-resolution vertical profiles of atmospheric aerosols and clouds to enable a greater understanding of our climate (Winker et al., 2004).

\section{Differential Absorption Lidar}

Differential Absorption Lidar (DIAL) is a laser remote sensing technique that is used for range and/or profile measurements of atmospheric gas concentrations and constituents (Ismail and Browell, 2015; Kramer, 2002).

\section{Spaceborne Laser Scanning}

Spaceborne Laser Scanning (e.g. Geoscience Laser Altimeter System - GLAS, Global Ecosystem Dynamics Investigation GEDI) provides mainly global measurements of the Earth's surface with the potential of capturing additional clouds and atmospheric aerosols. The spaceborne measurements allow to globally observe ice sheet and land elevations, approximate sea ice thickness, changes in elevation through time, vegetation coverage for biomass estimation, and height profiles of clouds and aerosols (Brenner et al., 2011).

\section{Airborne Laser Scanning}

Airborne Laser Scanning (ALS) systems allow a direct and illumination-independent measurement from $3 \mathrm{D}$ objects in a fast, remote and accurate way. Beside basic range measurements, the current commercial ALS developments allow to record the waveform of the backscattered laser pulse. Latest trends in sensor developments focus on single-photon detection. Different applications are of interest, like urban planning, forestry surveying, or power line monitoring. Further to describe the 3D scene, products like Digital Terrain Models (DTMs), Digital Surface Models (DSMs), or city models are provided (Mandlburger and Jutzi, 2018; Stilla and Jutzi, 2018).

\section{Terrestrial Laser Scanning}

A Terrestrial Laser Scanning (TLS) system is a stationary highly accurate ranging device for geodetic surveying. More specifically, TLS systems provide dense and accurate 3D point cloud data for the local environment and they may also reliably measure distances of several tens of meters. Due to these capabilities, such TLS systems are commonly used for applications such as city modelling, construction surveying, scene interpretation, urban accessibility analysis, or the digitization of cultural heritage objects. When using a TLS system, each captured TLS scan is represented in the form of a $3 \mathrm{D}$ point cloud consisting of a large number of scanned $3 \mathrm{D}$ points and, optionally, additional attributes for each $3 \mathrm{D}$ point such as color or intensity information. However, a TLS system represents a line-of-sight instrument and hence occlusions resulting from objects in the scene may be expected as well as a significant variation in point density between close and distant object surfaces. Thus, a single scan might not be sufficient in order to obtain a dense and (almost) complete 3D acquisition of interesting parts of a scene and, consequently, multiple scans have to be acquired from different locations (Barnea and Filin, 2012; Weinmann, 2016).

\section{Mobile Laser Scanning}

A Mobile Laser Scanning (MLS) system consists of a moving vehicle equipped with one or more usually side-looking laser scanners to capture information about the local 3D geometry (Weinmann et al., 2015; Paparoditis et al., 2012).

\section{Underwater Laser Scanning}

Underwater Laser Scanning is applied in deep-sea as well as in shallow water regions. The ranging distance is close range and the measurement principle relies on triangulation by laser light, comparable with structured-light-projection. More recently, companies started to develop Time-of-Flight (ToF) underwater laser scanners (Bleier et al., 2019).

\section{Bathymetric Laser Scanning}

For Bathymetric Laser Scanning the utilized green laser light with its potential penetration capabilities in water is essential. For water surface mapping the electromagnetic radiation of the laser penetrates into the topmost layer of the water column and can also be used for mapping the water surface and shallow water bathymetry. Area-wide water surface heights and depths are required for many disciplines such as hydrology, hydraulic engineering, flood risk management, ecology, climate change, etc. (Mandlburger and Jutzi, 2019).

\subsection{Related Skills}

Whilst the knowledge representation follows a thematic hierarchy, the skill description for each concept follows a different definition. In general, each concept of the EO4GEO BoK will have different skills, defined in order to correspond to different EQF (European Qualification Framework) levels. As such, for the definition of curricula or job descriptions, skills of a particular EQF level and their corresponding concepts can be selected, relating the concepts with each other.

As mentioned in Section 3.3., the skills related to the active optical sensors have been defined according to a six level taxonomy (Hofer et al., 2020) which ranges from more abstract verbs, relevant for describing e.g. course objectives (lower taxonomy levels), to more concrete verbs, relevant for task definition in e.g. job offers (higher taxonomy levels).

A few examples of defined skills for the different concept levels are summarized below.

\section{Skills for Level-1 Concepts}

Explain and discuss the LiDAR technology (taxonomy 2)

Select relevant LiDAR datasets (taxonomy 3 )

Analyse and classify LiDAR data (taxonomy 4)

Process and transform LiDAR data (taxonomy 6 )

Define the principles of operation of the structuredlight-projection camera and relate to stereoscopic measurements (taxonomy 1 ) 


\section{Skills for Level-2 Concepts}

- Explain the laser scanner technology (taxonomy 2)

- Compare different types of laser scanners (taxonomy 2)

- Understand and discuss the difference between laser scanners and laser profilers (taxonomy 2)

- Process and produce laser-profiler derivate products (taxonomy 6)

- Explain the principles of operation of a ranging camera (taxonomy 2)

- Compare the principles of operation of the multitemporal and multi-spectral pattern based sensors (taxonomy 2)

- Use a ranging camera (taxonomy 3)

- Interpret the IR image produced by speckle-pattern based sensor (taxonomy 5 )

- Evaluate and validate multi-temporal pattern based sensor products (taxonomy 5 )

\section{Skills for Level-3 Concepts}

Teach what information is acquired by the laser altimeters (taxonomy 3 )

- Identify the differences between a Wind Doppler LiDAR and a Differential Absorption LiDAR and select the appropriate data for a given application (taxonomy 3)

- Explain the principles of spaceborne laser scanning operation and discuss its applications (taxonomy 2)

- Choose the appropriate laser scanning device or data according to a given application (taxonomy 3 )

- Examine terrestrial laser scanning data with regard to occlusions (taxonomy 4)

- Analyse and classify underwater laser scanning data (taxonomy 4)

- $\quad$ Order bathymetric laser scanning data (taxonomy 4)

- Interpret the waveform of airborne laser scanning measurements (taxonomy 5)

- Create spaceborne laser scanning derivated products (taxonomy 6)

- $\quad$ Process mobile laser scanning data (taxonomy 6)

While skills at low taxonomy levels reflect general knowledge, skills at higher taxonomy levels focus additionally on the application and details of specific sensors, describing more demanding and specific skills that may be useful when defining a job offer or curricula.

\section{CONCLUSIONS AND OUTLOOK}

In this paper, we presented an excerpt of the EO4GEO BoK dedicated to active optical sensors. The structure of the 17 identified concepts was based on sensors, which in turn were linked to the relevant concepts on platforms, data, their processing and applications. The definition of the concepts was based on expert knowledge in the field. At the moment, five concepts are fully described and twelve have a short description. Subsequent work will include a full description of the missing concepts, the definition of additional job oriented skills and the addition of new relationships with other parts of the BoK. Some concepts, for which a full description seems redundant, may be concatenated into their super-concept.

The presented concepts constitute an important basis for the definition of learning paths for module catalogues as well as for the organisation of training actions and materials.

In order for this BoK to provide reliable and up-to-date information for the conception of curricula or job offers, it needs to be regularly updated and fed with new concepts representing the latest state-of-the-art. The first complete version of the EO4GEO BoK is expected in July 2021, until then and after experts, i.e., practitioners and researchers in the field of EO*GI, can register to the EO4GEO network of experts (Lemmens et al., 2020b) and improve and expand concepts and their definition. Moreover, the EO4GEO BoK will be integrated and linked with other relevant BoKs, so that more users and experts can contribute to its sustainable development.

\section{ACKNOWLEDGEMENTS}

The EO4GEO project would like to thank the scientists and specialists from the EO*GI sector who have already registered as EO4GEO experts and support the development of EO4GEO BoK, ensuring the completeness and highest professional standards of its content.

The project is co-funded by the Erasmus + Programme of the European Union Sector Skills Alliances N 591991-EPP-1-20171-IT-EPPKA2-SSA-B.

\section{REFERENCES}

Aguilar-Moreno, E., Casteleyn, S., Monfort-Muriach, A., 2020. Evolution of EO4GEO ecosystem of tools: beyond sector expectations. EO4GEO Newsletter. https://eo4geo.medium.com/evolution-of-eo4geoecosystem-of-tools-d9b1d061705 (20 January 2021).

Ahearn, S., Icke, I., Data, R., DeMers, M.N., Plewe, B., Skupin, A., 2013. Reengineering the GIS\&T Body of Knowledge. International Journal of Geographical Information Science, 27 (11) 2227-2245. doi.org/10.1080/13658816.2013.802324.

Barnea, S., Filin, S., 2012. Extraction of objects from terrestrial laser scans by integrating geometry image and intensity data with demonstration on trees. Remote Sensing, 4(1), 88-110. doi.org/10.3390/rs4010088.

Bethel, J., 2011. Imaging Body of Knowledge for the Professional Surveyor Surveying and Land Information Science, 71(3-4), 147-156(10).

Bleier, M., van der Lucht, J., Nüchter, A., 2019. Scout3D - An Underwater Laser Scanning System For Mobile Mapping. International Archives of the Photogrammetry, Remote Sensing and Spatial Information Sciences, XLII2/W18, 13-18. doi.org/10.5194/isprs-archives-XLII-2-W18-13-2019.

Brenner, A.C., Zwally, H.J., Bentley, C.R., Csatho, B.M., Harding, D.J., Hofton, M.A., Minster, J.B., Roberts, L.A., Saba, J.L., Thomas, R.H., Yi, D. 2011. Geoscience Laser Altimeter System (GLAS) - Derivation of Range and Range Distributions From Laser Pulse Waveform Analysis for Surface Elevations, Roughness, Slope, and Vegetation Heights. Algorithm Theoretical Basis Document-Version, 5. https://www.csr.utexas.edu/glas/pdf/WFAtbd_v5_02011Sept.pdf (20 January 2021).

Butterfield, A., Ngondi, G. E., \& Kerr, A. (Eds.). (2016). A dictionary of computer science. Oxford University Press. p.201

Dal Mutto, C., Zanuttigh, P., Cortelazzo, G. M., 2012. Time-of-Flight Cameras and Microsoft Kinect A User Perspective on Technology and Applications. Springer New York, USA. doi.org/10.1007/978-14614-3807-6.

DAMA International, 2017. DAMA-DMBOK: Data Management Body of Knowledge. 2nd Edition. Technics Publications.

DiBiase, D., DeMers, M., Johnson, A., Kemp, K., Luck, A. T., Plewe, B., Wentz, E., 2006. Geographic Information Science and Technology Body of Knowledge. Association of American Geographers and University Consortium for Geographic Information Science. Washington https://www.ucgis.org/assets/docs/gist_body_of_knowledge.pdf (20 January 2021).

Geng, J., 2011. Structured-light 3D surface imaging: a tutorial. Advances in Optics and Photonics, 3(2), 128-160. doi.org/10.1364/AOP.3.000128.

Greenfeld, J., Potts, L., 2008. Surveying body of knowledge-Preparing professional surveyors for the 21 st century. Surveying and Land Information Science, 68(3), 133-143. 
Greenfeld, J., 2010. Surveying Body of Knowledge. In FIG Congress, Sydney, Australia.

Greenfeld, J., 2011. Surveying body of knowledge. Surveying and Land Information Science, 71(3-4), 105-113.

Greenfeld, J., LS, I., 2012. Components of the Surveying body of knowledge. In FIG Working Week. Rome, Italy.

Hofer, B., Casteleyn, S., Missoni-Steinbacher, E.M., Aguilar-Moreno, E., Albrecht, F., Lemmens, R., Lang, S., Albrecht, J., Stelmaszczuk-Górska, M.A., Vancauwenberghe, G., Monfort-Muriach, A., 2020. From Tasks to Concepts and Skills - Complementing the Body of Knowledge with a Business-oriented Perspective. Transactions in GIS, 24 (3), 587-601, doi.org/10.1111/tgis.12628.

Ismail, S., Browell, E.V., 2015. LIDAR / Differential Absorption Lidar. Encyclopedia of Atmospheric Sciences (Second Edition). 277-288. Academic Press. doi.org/10.1016/B978-0-12-382225-3.00204-8.

Jutzi, B., 2009. Investigations on ambiguity unwrapping of range images. International Archives of Photogrammetry and Remote Sensing, 38 (3/W8), 265-270.

Jutzi, B., 2015. Methoden zur automatischen Szenencharakterisierung basierend auf aktiven optischen Sensoren für die Photogrammetrie und Fernerkundung. Habilitation.

https://publikationen.bibliothek.kit.edu/1000050691 (20 January 2021).

Jutzi, B., Meyer, F., Hinz, S., 2016. Aktive Fernerkundungssensorik Technologische Grundlagen und Abbildungsgeometrie. In: Freeden, W., Rummel, R. (Hrsg) Handbuch der Geodäsie. Springer Berlin Heidelberg, Germany. doi.org/10.1007/978-3-662-46900-2_40-1.

Keranen, K., Kolvoord, R., 2019. Light Detection and Ranging (LiDAR). The Geographic Information Science \& Technology Body of Knowledge (4th Quarter 2019 Edition), John P. Wilson (ed.) doi.org/10.22224/gistbok/2019.4.17.

Kramer, H.J., 2002. Observation of the Earth and its Environment: Survey of Missions and Sensors. Springer-Verlag Berlin Heidelberg, Germany. doi.org/10.1007/978-3-642-56294-5.

Kreiger, V., 2020. Innovative Training Actions - Applying the EO4GEO methods \& findings in case-based approaches. EO4GEO Newsletter. https:/eo4geo.medium.com/innovative-training-actions-eo4geo-learningmethod-1f0e47c6f58d (20 January 2021).

Lemmens, R.L.G., Ronzhin, S., Augustijn, P.W.M., Verkroost, M.J., Walsh, N., 2018. Space Education with The Living Textbook, A web-based tool using a Concept Browser. Paper presented at 2nd Symposium on Space Educational Activities, SSEA 2018, Budapest, Hungary.

Lemmens, R.L.G., Hoscilo, A., Aguilar-Moreno, E. M., StelmaszczukGórska, M., Olijslagers, M., 2020a. Become an EO4GEO expert, contribute and be acknowledged as BoK developer! EO4GEO Newsletter https://eo4geo.medium.com/become-an-eo4geo-expert-contribute-and-beacknowledged-as-bok-developer-7b21 fd1e262 (20 January 2021).

Lemmens, R.L.G., Olijslagers, M., Stelmaszczuk-Górska, M., 2020b. EO4GEO Tools Manual - Viewing the public EO4GEO BoK with the Living Textbook (LTB). Version 1.5 . https://docs.google.com/document/d/1gSTIkA1iSq0X6LOX95Z8GseHlyWb Pa4IleRfTnWCVyI/edit?usp=sharing (20 January 2021).

Mandlburger, G., Jutzi, B., 2018. Feasibility Investigation on Single Photon LIDAR Based Water Surface Mapping. ISPRS Annals of the Photogrammetry, Remote Sensing and Spatial Information Sciences, IV-1, 109-116. doi.org/10.5194/isprs-annals-IV-1-109-2018.

Mandlburger, G., Jutzi, B., 2019. On the feasibility of water surface mapping with single photon lidar. ISPRS International Journal of Geo-Information, 8(4), 188. doi.org/10.3390/ijgi8040188.

Paparoditis, N., Papelard, J.P., Cannelle, B., Devaux, A., Soheilian, B., David, N., Houzay, E., 2012. Stereopolis II: A multi-purpose and multi-sensor 3D mobile mapping system for street visualisation and 3D metrology. Revue Française de Photogrammétrie et de Télédétection, 200(1), 69-79.

PMI, 2017. A Guide to the Project Management Body of Knowledge: $P M B O K{ }^{\circledR}$ Guide. 6th edition. Project Management Institute.

Rees, W.G., 2013. Physical principles of remote sensing. Cambridge University Press.
Reinhardt, W., 2014. Zur Bedeutung eines „Body of Knowledge“ für die Geoinformatik. Publikationen der Deutschen Gesellschaft für Photogrammetrie, Fernerkundung und Geoinformation, 23(133), 7. ISSN: 0942-2870.

Rickles, P., Ellul, C., Haklay, M., 2017. A Suggested Framework and Guidelines for Learning GIS in Interdisciplinary Research. Geo: Geography and Environment, 4(2), 1-18. doi.org/10.1002/geo2.46.

Riveiro, B., Lindenbergh, R. (Eds.), 2019. Laser Scanning: An Emerging Technology in Structural Engineering (Vol. 14). CRC Press.

Salvi, J., Pages, J., Batlle, J., 2004. Pattern codification strategies in structured light systems. Pattern Recognition, 37(4), 827-849. doi.org/10.1016/j.patcog.2003.10.002.

Shan, J., Toth, C.K. (Eds.), 2018. Topographic laser ranging and scanning: principles and processing. CRC press.

Stelmaszczuk-Górska, M., Aguilar-Moreno, E., Casteleyn, S., Vandenbroucke, D., Miguel-Lago, M., Dubois, C., Lemmens, R. Vancauwenberghe, G., Olijslagers, M., Lang, S., Albrecht, F., Belgiu, M., Krieger, V., Jagdhuber, T., Fluhrer, A., Soja, M.J., Mouratidis, A., Persson, H., Colombo, R., Masiello, G., 2020a. Body of Knowledge for the Earth Observation and Geoinformation Sector - A Basis for Innovative Skills Development. International Archives of the Photogrammetry, Remote Sensing and Spatial Information Sciences, XLIII-B5-2020, 15-22. doi.org/10.5194/isprs-archives-XLIII-B5-2020-15-2020.

Stelmaszczuk-Górska, M., Aguilar-Moreno, E., Casteleyn, S., Lemmens, R., 2020b. Body of Knowledge for Earth Observation and Geographic Information is publicly available! - What you can expect from it? EO4GEO Newsletter. https://eo4geo.medium.com/body-of-knowledge-forearth-observation-and-geographic-information-is-publicly-available$7 \mathrm{a} 37 \mathrm{dbd} 42 \mathrm{ad} 0$ (20 January 2021).

Stilla, U., Jutzi, B., 2018. Full-waveform analysis for pulsed laser systems. In Shan J., Toth C.K. (Eds.) Topographic Laser Ranging and Scanning: Principles and Processing (2nd Edition). 239-258, CRC Press, Boca Raton. ISBN 978-1-4200-5142-1.

Sudmanns, M., Tiede, D., Lang, S., Bergstedt, H., Trost, G., Augustin, H., Baraldi, A., Blaschke, T., 2019. Big Earth data: disruptive changes in Earth observation data management and analysis? International Journal of Digital Earth, 1-19. doi.org/10.1080/17538947.2019.1585976.

Vandenbroucke, D., Vancauwenberghe, G., 2016. Towards a New Body of Knowledge for Geographic Information Science and Technology. Micro, Macro \& Mezzo Geoinformation, 2016 (6), 7-19. ISSN: 1857-9000, EISSN 1857-9019.

Vandenbroucke, D., Gruijthuijsen, W., Olijslagers, M. Vancauwenberghe, G., Monfort Muriach, A., Casteleyn, S., Aguilar, E., Lemmens, R., StelmaszczukGórska, M., 2019. D2.2 - Revised ontology-based approach. Report of the EO4GEO Project funded with the support of the Erasmus + Programme of the European Union Sector Skills Alliances $N^{\circ}$ 591991-EPP-1-2017-1-ITEPPKA2-SSA-B. http://www.eo4geo.eu/download/eo4geo d-2-2-revisedontology-based-approach_v2-0/?wpdmdl $=3491 \&$ masterkey $=\overline{5 \mathrm{c}} 5 \mathrm{~b} 01 \mathrm{~d} 87 \mathrm{~d} 8 \mathrm{bd}$ (20 January 2021).

Vosselman, G., Maas, H.G., 2010. Airborne and terrestrial laser scanning. Whittles Publishing. ISBN 978-1904445-87-6.

Weinmann, M., Jutzi, B., Hinz, S., Mallet, C., 2015. Semantic point cloud interpretation based on optimal neighborhoods, relevant features and efficient classifiers. ISPRS Journal of Photogrammetry and Remote Sensing, 105, 286304. doi.org/10.1016/j.isprsjprs.2015.01.016.

Weinmann, M., 2016. Reconstruction and Analysis of 3D Scenes - From Irregularly Distributed 3D Points to Object Classes. Springer International Publishing. ISBN 978-3-319-29246-5.

Wilson, J.P. (ed.) 2020. The Geographic Information Science \& Technology Body of Knowledge. ISSN 2577-2848.

https://gistbok.ucgis.org/ (20 January 2021)

Winker, D. M., Hunt, W. H., Hostetler, C.A., 2004. Status and performance of the CALIOP lidar. Laser Radar Techniques for Atmospheric Sensing of the International Society for Optics and Photonics, 5575, 8-15. doi.org/10.1117/12.571955. 